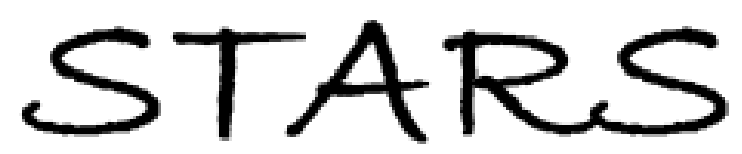

University of Central Florida

STARS

$1-1-2013$

\title{
Angular dependent reflections of a monodomain blue phase liquid crystal
}

Jin Yan

University of Central Florida

Yuan Chen

University of Central Florida

Daming Xu

University of Central Florida

Shin-Tson Wu

University of Central Florida

Find similar works at: https://stars.library.ucf.edu/facultybib2010 University of Central Florida Libraries http://library.ucf.edu

This Article is brought to you for free and open access by the Faculty Bibliography at STARS. It has been accepted for inclusion in Faculty Bibliography 2010 s by an authorized administrator of STARS. For more information, please contactSTARS@ucf.edu.

\section{Recommended Citation}

Yan, Jin; Chen, Yuan; Xu, Daming; and Wu, Shin-Tson, "Angular dependent reflections of a monodomain blue phase liquid crystal" (2013). Faculty Bibliography 2010 s. 4883.

https://stars.library.ucf.edu/facultybib2010/4883

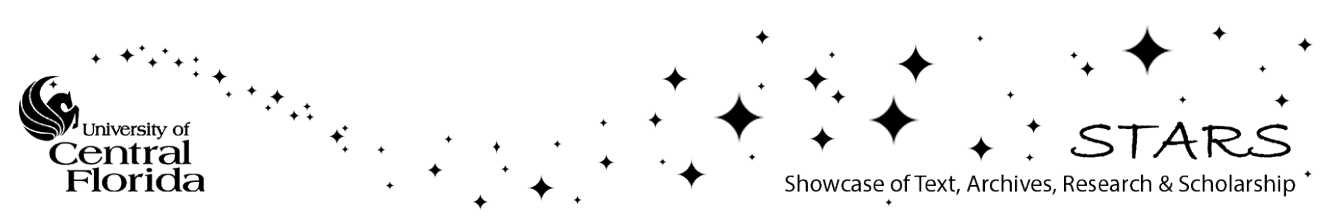




\section{Angular dependent reflections of a monodomain blue phase liquid crystal}

Cite as: J. Appl. Phys. 114, 113106 (2013); https://doi.org/10.1063/1.4821968

Submitted: 17 August 2013 . Accepted: 06 September 2013 . Published Online: 18 September 2013

Jin Yan, Yuan Chen, Daming Xu, and Shin-Tson Wu
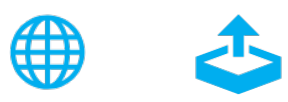

\section{ARTICLES YOU MAY BE INTERESTED IN}

Low voltage and high contrast blue phase liquid crystal with red-shifted Bragg reflection Applied Physics Letters 102, 011113 (2013); https://doi.org/10.1063/1.4773985

Electric field-induced monodomain blue phase liquid crystals

Applied Physics Letters 102, 171110 (2013); https://doi.org/10.1063/1.4803922

Low voltage blue-phase liquid crystal displays

Applied Physics Letters 95, 231101 (2009); https://doi.org/10.1063/1.3271771

\section{Applied Physics Reviews} Now accepting original research

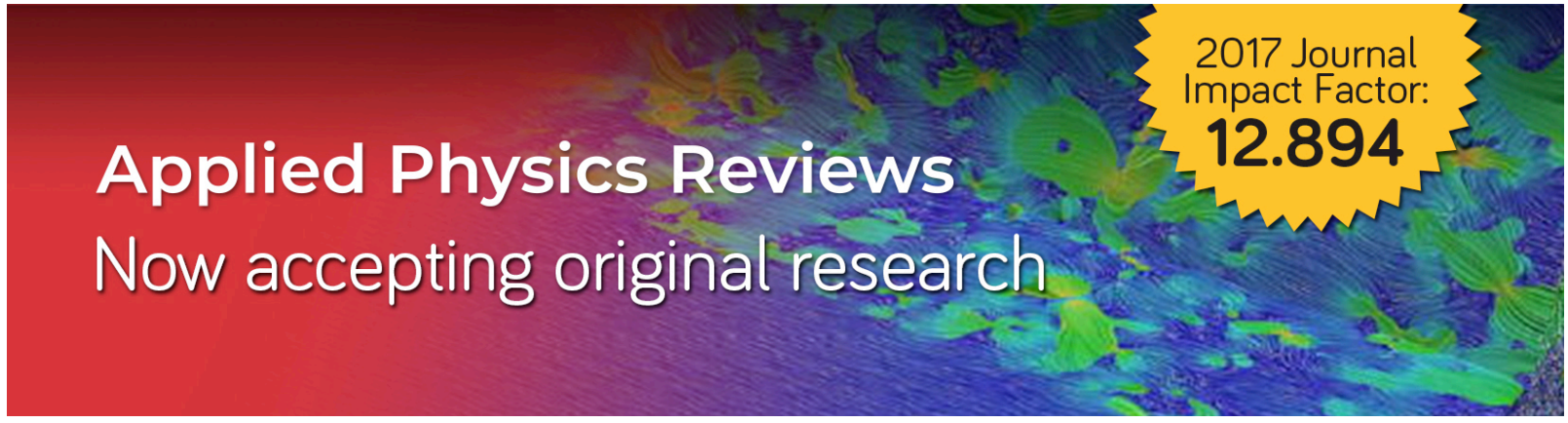




\title{
Angular dependent reflections of a monodomain blue phase liquid crystal
}

\author{
Jin Yan, Yuan Chen, Daming $\mathrm{Xu}$, and Shin-Tson $\mathrm{Wu}^{\mathrm{a})}$ \\ College of Optics and Photonics, University of Central Florida, Orlando, Florida 32816, USA
}

(Received 17 August 2013; accepted 6 September 2013; published online 18 September 2013)

\begin{abstract}
The angular dependent reflection of a monodomain blue phase liquid crystal (BPLC) is investigated. Unlike a cholesteric liquid crystal with single twist structure, a monodomain BPLC exhibits several reflection orders from different crystal planes. As incident angle increases, the first order reflection experiences a blue shift while the second order experiences a red shift. We analyze the physical mechanism of the reflection from six (110) crystal planes. Good agreement between calculated and experimental results is obtained. Undoubtedly, this angular dependency would affect the performance of BPLC photonic devices that require an oblique incidence. (C) 2013 AIP Publishing LLC.

[http://dx.doi.org/10.1063/1.4821968]
\end{abstract}

\section{INTRODUCTION}

Polymer-stabilized blue phase liquid crystal (PSBPLC) ${ }^{1-4}$ exhibits great potential for photonic ${ }^{5-7}$ and display ${ }^{8-12}$ applications. The most obvious feature for BPLC is the selective Bragg reflection resulting from the selfassembled cubic structure. ${ }^{13}$ For transmissive displays, the reflection band is intentionally shifted to ultraviolet region in order to realize a high contrast transparent display. A transmissive BPLC exhibit advantages of submillisecond response time, ${ }^{14,15}$ alignment layer free, optical isotropy to enable intrinsic wide viewing angle, and cell gap insensitive when in-plane-switching (IPS) mode is used. ${ }^{9}$ However, the main challenge is high driving voltage. To reduce voltage, several approaches have been proposed, such as protruded electrodes, ${ }^{9}$ vertical field switching, ${ }^{10,16}$ large Kerr constant materials, ${ }^{17,18}$ and red-shifting the reflection band to $\sim 600 \mathrm{~nm}$ while using circular polarizers to maintain high contrast ratio. ${ }^{19}$ Reflective displays with monodomain BPLC have also been demonstrated with either surface alignment method $^{20}$ or by applying an electric field during the cooling process. $^{21}$ The narrow bandwidth of Bragg reflection provides vivid colors for reflective displays. Amplitude modulation is realized by applying an electric field to change the local refractive index. The selective reflection feature also finds applications in photonic devices, such as lasing ${ }^{22,23}$ and tunable colors. $^{24,25}$ For applications requiring oblique incident angles, the angular dependency of Bragg reflection in BPLC is a critical factor, which remains unexplored.

In this paper, we investigate the angular dependency of a monodomain blue phase liquid crystal. Unlike a cholesteric liquid crystal with single twist structure, a monodomain BPLC has several reflections from different crystal planes. As incident angle increases, the first order reflection has a blue shift while the second order has a red shift. For a fixed incident angle, the reflection wavelength is also azimuthal angle dependent. We analyzed the physical mechanism of the reflection from six (110) crystal planes. Good agreement between calculation and experimental results is obtained.

\section{EXPERIMENTAL}

In experiment, we prepared a monodomain PS-BPLC with surface alignment. The nematic LC host used in this study is HTG-135200 (HCCH, China). It has following physical properties: $\Delta \mathrm{n}=0.2$ at $\lambda=633 \mathrm{~nm}, \Delta \varepsilon=96$ at $1 \mathrm{kHz}$ and $22^{\circ} \mathrm{C}$, and clearing temperature $\mathrm{T}_{\mathrm{c}}=97^{\circ} \mathrm{C}$. To form a BPLC precursor, we mixed the LC host with 2.86 wt. \% high twisting power chiral dopant S5011 (HTP $\approx 126 / \mu \mathrm{m}, \mathrm{HCCH})$, 10 wt. \% monomers [6 wt. \% RM257 (Merck) and 4 wt. \% TMPTA (1,1,1-Trimethylolpropane Triacrylate, Sigma Aldrich)], and 0.6 wt. $\%$ photoinitiator. Afterwards, the BPLC precursor was injected to a $90^{\circ}$ twisted nematic cell with orthogonal rubbings on the inner side of the substrates. The cell was slowly cooled down from isotropic phase at $1{ }^{\circ} \mathrm{C} / \mathrm{min}$ and hold at BPI at $70.5^{\circ} \mathrm{C}$. A uniform colored texture appears because the surface alignment helps create monodomain blue phase. ${ }^{20,26,27}$ The cell was then exposed to UV light $(\lambda=365 \mathrm{~nm})$ with an intensity of $2 \mathrm{~mW} / \mathrm{cm}^{2}$ for $30 \mathrm{~min}$. After UV irradiation, a monodomain PS-BPLC was formed.

Figure 1 depicts the experimental setup for measuring the angular dependent transmission spectra of the prepared monodomain PS-BPLC. The white light was filtered by an iris and collimated by a lens system. Here, we used a single lens to represent the lens system. The BPLC sample can be rotated around the vertical axis so that light is incident on the cell with different incident angles. The transmitted light was then collected by a focusing lens system and analyzed by a spectrometer (HR2000 CG-UV-NIR, Ocean Optics).

Figure 2 shows the measured transmission spectra for different incident angles. At normal incidence $\left(\theta=0^{\circ}\right)$, there

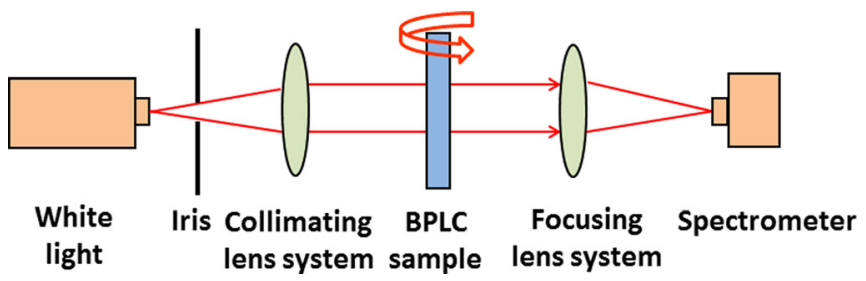

FIG. 1. Optical setup for measuring the angular dependent spectrum of a monodomain PS-BPLC. A lens system is represented by a single lens.

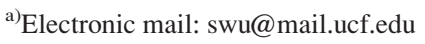




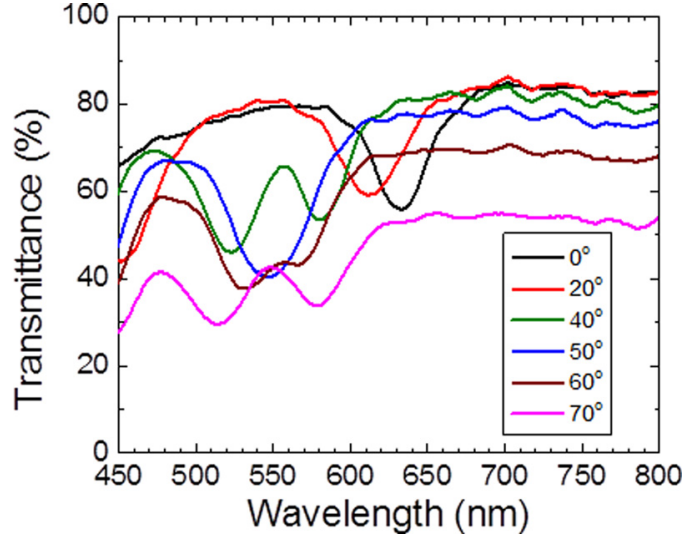

FIG. 2. Measured transmission spectra at different incident angles for a monodomain PS-BPLC. Light is incident from air. T $=23^{\circ} \mathrm{C}$.

is a reflection band at $\lambda \sim 630 \mathrm{~nm}$. As the incident angle increases, this reflection band shifts to a shorter wavelength. It is understandable since Bragg reflection usually has a blue shift at oblique incident angle. However, it is interesting to notice that there is a second reflection band which shifts to a longer wavelength as the incident angle increases. At $\theta=50^{\circ}$, these two reflection bands overlap at $\sim 550 \mathrm{~nm}$.

\section{THEORY AND DISCUSSION}

To explain the observed transmission spectra, we investigated the underlying physical mechanism. For a cholesteric liquid crystal with planar alignment, the reflection wavelength $\lambda_{0}=n \cdot P$, where $n$ is the average refractive index and $P$ is the pitch length. The reflection wavelength is simply related to incident angle as

$$
\lambda=\lambda_{0} \cos \theta
$$

where $\lambda_{0}$ is the reflection wavelength at normal incidence and $\theta$ is the incident angle. Different from cholesteric liquid crystals, the reflection wavelength of a blue phase liquid crystal depends on the corresponding crystal plane. For a light normally incident on a crystal plane, the reflection wavelength can be expressed as ${ }^{28}$

$$
\lambda_{0}=\frac{2 n a}{\sqrt{h^{2}+k^{2}+l^{2}}},
$$

where $n$ and $a$ denote average refractive index and lattice constant of blue phases, and $h, k$, and $l$ are the Miller indices of the crystal plane. In BPI, the lattice constant $a=P$. For a monodomain BPI with body-centered cubic (bcc) structure, the crystal plane (110) is parallel to the substrate since it is the most densely packed of bcc crystal structures and is therefore the lowest energy surface. However, there are six planes in the (110) group: $(101),(10 \overline{1}),(110),(1 \overline{1} 0),(011)$, $(01 \overline{1})$, as indicated by red, blue, and green colors in Fig. 3. Here, we assume (101) plane is parallel to the substrate. It will not make a difference if we select another plane in the (110) group because of symmetry. We also assume $(10 \overline{1})$ plane is the incident plane with azimuthal angle $\phi=0$. For different incident angles $\theta$ in the (10 $\overline{1})$ incident plane, we

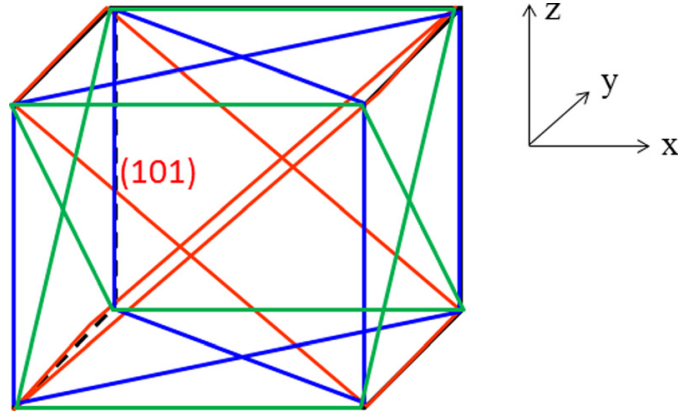

FIG. 3. Six (110) planes indicated by red, green and blue colors in the cubic structure of a BPI. The crystal plane (101) is parallel to the substrate, indicated by red lines.

calculated the angles between incident light and the normal of six planes in (110) group, listed in Table I. For the light normally incident on (101) plane, according to Eq. (2), its reflection wavelength should be $\lambda_{0}=\sqrt{2} n a$. Assuming the refractive index of BPLC is 1.58, we find the lattice constant $a$ is $\sim 282 \mathrm{~nm}$. This normally incident light, however, has $60^{\circ}$ incident angle with respect to $(110),(1 \overline{1} 0),(011),(01 \overline{1})$ planes and the reflection wavelength can be expressed as

$$
\lambda=\lambda_{0} \cos \theta^{\prime}
$$

where $\lambda_{0}$ is the reflection wavelength at normal incidence and $\theta^{\prime}$ is the incident angle with respect to a crystal plane, in order to distinguish with the incident angle $\theta$. As $\theta$ increases, the angle $\theta^{\prime}$ for (101) plane increases, while $\theta^{\prime}$ for (011) and (110) planes decreases. Therefore, the first order reflection has a blue shift with oblique incident angle, while the second and the third order reflections have a red shift. The observed reflection orders cannot come from different crystal plane group such as (200) since the longest wavelength for (200) plane is $\sim 445 \mathrm{~nm}$ according to Eq. (2).

To validate our theory, we first measured the azimuthal angle dependency at a fixed incident angle $\theta=40^{\circ}$. Figure 4 depicts the reflection wavelengths of the first three reflection orders at different azimuthal angles. For some azimuthal angles, we are able to observe the third reflection order. The circles are experimental data and the lines are calculated results. Here, we define the first order as the longest reflection wavelength at normal incidence, which is the reflection from (101) plane. The second order and the third order are also defined according to the reflection wavelength but the

TABLE I. Calculated angles between incident light and the normal direction of six crystal planes in (110) group for different incident angles. The incident plane is $(10 \overline{1})$. Light is incident from the air. The refractive index of BPLC is 1.58 .

\begin{tabular}{lrrrrrr}
\hline \hline Incident angle & $(101)$ & $(10 \overline{1})$ & $(011)$ & $(01 \overline{1})$ & $(110)$ & $(1 \overline{1} 0)$ \\
\hline 0 & 0.0 & 90.0 & 60.0 & 60.0 & 60.0 & 60.0 \\
10 & 6.3 & 90.0 & 54.9 & 65.2 & 54.9 & 65.2 \\
20 & 12.5 & 90.0 & 50.1 & 70.4 & 50.1 & 70.4 \\
30 & 18.4 & 90.0 & 45.7 & 75.5 & 45.7 & 75.5 \\
40 & 24.0 & 90.0 & 41.9 & 80.3 & 41.9 & 80.3 \\
50 & 29.0 & 90.0 & 38.7 & 84.6 & 38.7 & 84.6 \\
60 & 33.2 & 90.0 & 36.3 & 88.2 & 36.3 & 88.2 \\
\hline \hline
\end{tabular}




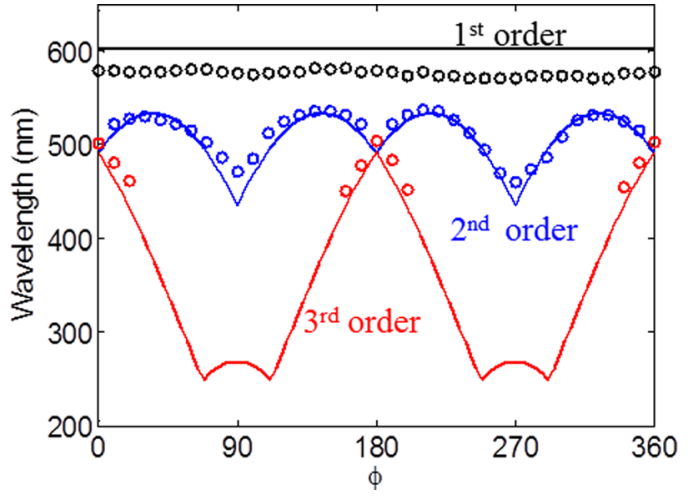

FIG. 4. Measured (circles) and simulated (lines) reflection wavelengths of first three reflection orders for $360^{\circ}$ azimuthal angles.

third order reflection does not necessarily come from the same crystal plane. In the simulation, there is only one adjustable parameter: the Bragg reflection wavelength $\lambda_{0}$ for normal incidence and we used $\lambda_{0}=660 \mathrm{~nm}$, which is slightly different from the measured value $630 \mathrm{~nm}$. The fitting is very good for the 2 nd and 3rd orders while there is a small mismatch for the 1 st order. It is reasonable by considering that (101) planes are confined along the cell gap direction so the lattice is slightly distorted because of the cell gap confinement.

Next, we studied the incident angle dependency. To obtain a larger tunable range, we immersed the cell into water so that the angle inside the cell is larger compared to the air environment because of Snell's Law. ${ }^{10}$ We chose two orthogonal incident planes to study the incident angle effect. The two incident planes are parallel to the edges of the rectangular BPLC cell. The azimuthal angles for these two incident planes happen to be $24^{\circ}$ and $114^{\circ}$ if we follow the abovementioned definition. Figures 5(a) and 5(b) show the measured (dots) and simulated (lines) results of the first three reflection orders for two incident planes, respectively. In the simulation, we used the same $\lambda_{0}=660 \mathrm{~nm}$ and azimuthal angles $\phi=24^{\circ}$ and $114^{\circ}$. There is no adjustable parameter. From Fig. 5, the calculated results match well with the experimental data. Therefore, our theory is validated. Moreover, the reflection wavelength almost covers the entire visible region which could be useful for display and photonic applications.

Although there are several reflection orders from the monodomain BPLC cell, not all the reflected light can exit from the cell. Figure 6 depicts two photos of the BPLC cell. The cell is immersed in water and light is incident on the cell with a fixed angle $\theta \sim 50^{\circ}$. We moved the camera to take pictures from two different angles. Figure 6(a) reflects green light, which is the first order reflection. By moving the camera to a different direction, we are able to see a vivid red color as shown in Fig. 6(b) which originates from the second order reflection. However, we are unable to collect this reflected light by a detector. If the reflected light is incident on the glass substrate with an angle larger than the critical angle, it will experience TIR (total internal reflection) and cannot exit from the cell. This feature could make the monodomain BPLC an attractive candidate for waveguide applications.
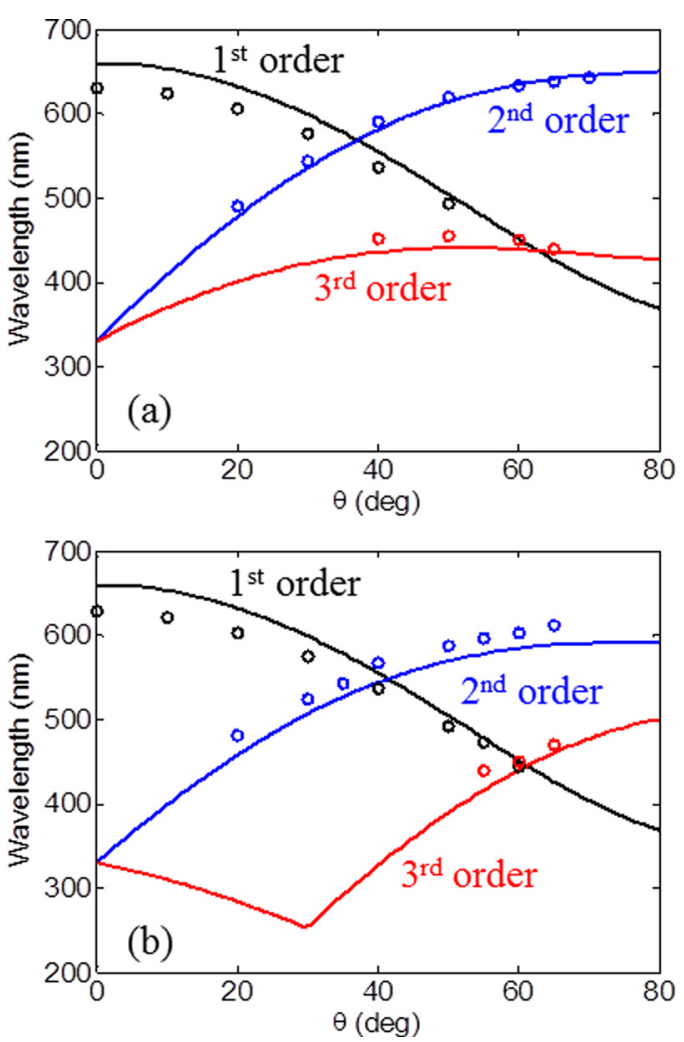

FIG. 5. Measured (circles) and simulated (lines) reflection wavelengths of first three reflection orders for different incident angles at (a) $\phi=24^{\circ}$ and (b) $\phi=114^{\circ}$.
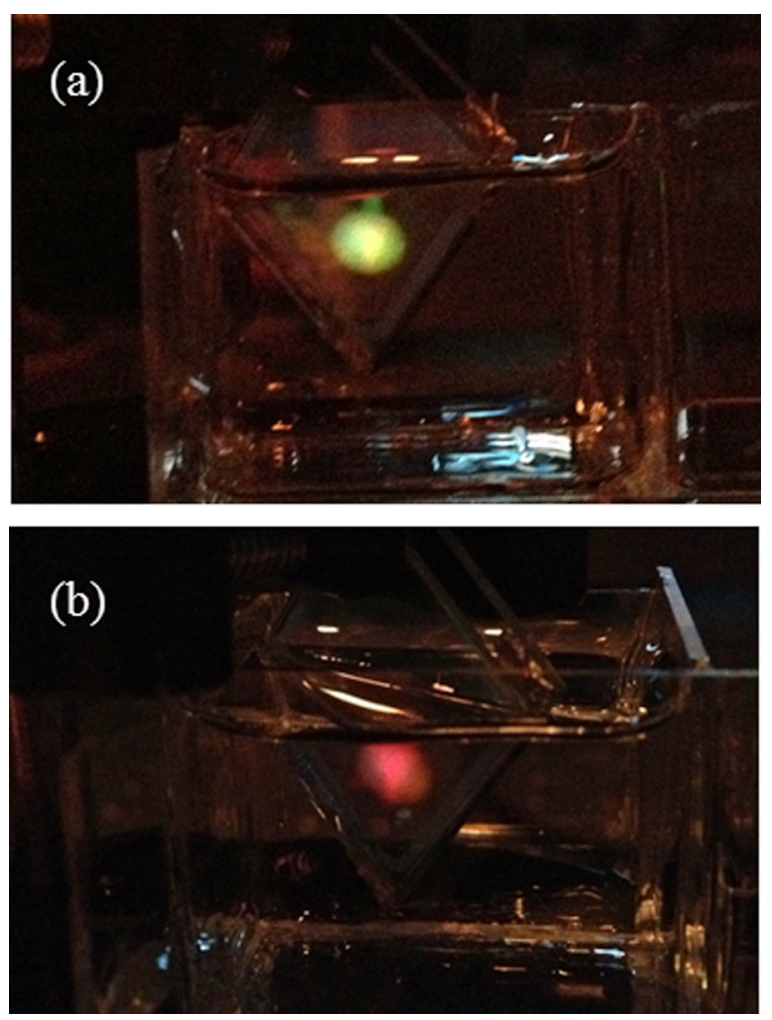

FIG. 6. (a) First order reflection and (b) second order reflection of a BPLC cell. Cell is immersed in water and light is incident at $\theta \sim 50^{\circ}$. Two photos are taken at different angles. 


\section{CONCLUSION}

In conclusion, we have investigated both polar and azimuthal angle dependencies of a monodomain PS-BPLC sample. The cubic structure of PS-BPLC makes its angular dependency quite different from a cholesteric LC. We analyzed the physical mechanism of the observed three reflection orders. According to our proposed mechanism, we further predicted reflection wavelengths for different incident planes and incident angles. The predicted results match well with the measured results. The angular dependency of PSBPLC is an important factor in display and photonic applications. It also shows other potential applications such as tunable colors and waveguides.

\section{ACKNOWLEDGMENTS}

The authors would like to thank Industrial Technology Research Institute (ITRI, Taiwan) for financial support.

${ }^{1}$ H. Kikuchi, M. Yokota, Y. Hisakado, H. Yang, and T. Kajiyama, Nature Mater. 1, 64 (2002)

${ }^{2}$ Y. Haseba, S. Yamamoto, K. Sago, A. Takata, and H. Tobata, SID Int. Symp. Digest Tech. Papers 44, 254 (2013).

${ }^{3}$ J. Yan, L. Rao, M. Jiao, Y. Li, H. C. Cheng, and S. T. Wu, J. Mater. Chem. 21, 7870 (2011)

${ }^{4}$ M. Wittek, N. Tanaka, M. Bremer, D. Pauluth, K. Tarumi, M. C. Wu, D. M. Song, and S. E. Lee, SID Int. Symp. Digest Tech. Papers 42, 292 (2011).

${ }^{5}$ Y. H. Lin, H. S. Chen, H. C. Lin, Y. S. Tsou, H. K. Hsu, and W. Y. Li, Appl. Phys. Lett. 96, 113505 (2010).

${ }^{6}$ J. Yan, Y. Li, and S. T. Wu, Opt. Lett. 36, 1404 (2011).

${ }^{7}$ Y. Li and S. T. Wu, Opt. Express 19, 8045 (2011).

${ }^{8}$ Z. Ge, S. Gauza, M. Jiao, H. Xianyu, and S. T. Wu, Appl. Phys. Lett. 94, 101104 (2009).
${ }^{9}$ L. Rao, Z. Ge, S. T. Wu, and S. H. Lee, Appl. Phys. Lett. 95, 231101 (2009).

${ }^{10}$ H. C. Cheng, J. Yan, T. Ishinabe, and S. T. Wu, Appl. Phys. Lett. 98, 261102 (2011).

${ }^{11}$ H. Lee, H. J. Park, O. J. Kwon, S. J. Yun, J. H. Park, S. Hong, and S. T. Shin, SID Int. Symp. Digest Tech. Papers 42, 121 (2011).

${ }^{12}$ Y. Hirakata, D. Kubota, A. Yamashita, T. Ishitani, T. Nishi, H. Miyake, H. Miyairi, J. Koyama, S. Yamazaki, T. Cho, and M. Sakakura, J. Soc. Inf. Disp. 20, 38 (2012).

${ }^{13}$ S. Meiboom, J. P. Sethna, P. W. Anderson, and W. F. Brinkman, Phys. Rev. Lett. 46, 1216 (1981).

${ }^{14}$ K. M. Chen, S. Gauza, H. Q. Xianyu, and S. T. Wu, J. Disp. Technol. 6, 49 (2010).

${ }^{15}$ Y. Chen, J. Yan, J. Sun, S. T. Wu, X. Liang, S. H. Liu, P. J. Hsieh, K. L. Cheng, and J. W. Shiu, Appl. Phys. Lett. 99, 201105 (2011).

${ }^{16}$ Y. H. Kim, S. T. Hur, C. S. Park, K. W. Park, S. W. Choi, S. W. Kang, and H. R. Kim, Opt. Express 19, 17427 (2011).

${ }^{17}$ L. Rao, J. Yan, S. T. Wu, S. Yamamoto, and Y. Haseba, Appl. Phys. Lett. 98, 081109 (2011)

${ }^{18}$ Y. Chen, D. Xu, S. T. Wu, S. Yamamoto, and Y. Haseba, Appl. Phys. Lett. 102, 141116 (2013).

${ }^{19}$ J. Yan, Z. Luo, S. T. Wu, J. W. Shiu, Y. C. Lai, K. L. Cheng, S. H. Liu, P. J. Hsieh, and Y. C. Tsai, Appl. Phys. Lett. 102, 011113 (2013).

${ }^{20}$ J. Yan, S. T. Wu, K. L. Cheng, and J. W. Shiu, Appl. Phys. Lett. 102, 081102 (2013).

${ }^{21}$ Y. Chen and S. T. Wu, Appl. Phys. Lett. 102, 171110 (2013).

${ }^{22}$ W. Cao, A. Munoz, P. Palffy-Muhoray, and B. Taheri, Nature Mater 1, 111 (2002).

${ }^{23}$ S. Yokoyama, S. Mashiko, H. Kikuchi, K. Uchida, and T. Nagamura, Adv. Mater. 18, 48 (2006).

${ }^{24}$ G. Heppke, B. Jerome, H. S. Kitzerow, and P. Pieranski, J. Phys. (France) 50, 2991 (1989).

${ }^{25}$ H. J. Coles and M. N. Pivnenko, Nature 436, 997 (2005).

${ }^{26}$ P. Nayek, H. Jeong, H. R. Park, S. W. Kang, S. H. Lee, H. S. Park, H. J. Lee, and H. S. Kim, Appl. Phys. Express 5, 051701 (2012).

${ }^{27}$ H. Y. Liu, C. T. Wang, C. Y. Hsu, and T. H. Lin, Appl. Opt. 50, 1606 (2011).

${ }^{28}$ H. Kikuchi, Liquid Crystalline Blue Phases, edited by Takashi Kato (Springer, Berlin, Heidelberg, 2008). 